\title{
A Method For Numerical Integration
}

\author{
By C. B. Haselgrove
}

1. Introduction. In this paper we shall give an account of some methods developed for the numerical evaluation of multidimensional integrals. These methods are based on the theory of Diophantine approximation. They are suitable for some problems for which the Monte Carlo method is commonly used and, like the Monte Carlo method, are well fitted for use with an electronic digital computer. We shall show, however, that they are superior to the Monte Carlo method provided that the integrand satisfies certain conditions. We shall also show that they are superior, for integrals in space of several dimensions, to formulas typified by those of Gauss and Simpson; they may be superior even to certain new integration formulas specially constructed for the evaluation of multiple integrals (see for example Hammer [2], who gives a bibliography, and Miller [5], [6], [7]).

The method of antithetic variates which is described by Hammersley and others [3], [4] may be used to obtain better estimates than the Monte Carlo method but the author thinks that the method described in the present paper is simpler to apply and gives better results.

Various authors have suggested methods which are particular cases of those described in this paper but without the underlying theory. See for example Davis and Rabinowitz [1].

In this section we shall give a short account of the behavior of the error in the Monte Carlo method and the direct-product Gauss-type methods so that we can compare these with the errors of the new methods. We shall not give an account of the method of antithetic variates.

Suppose that we wish to estimate the integral

$$
I=\int_{0}^{1} \int_{0}^{1} \cdots \int_{0}^{1} f\left(x_{1}, x_{2}, \cdots, x_{k}\right) d x_{1} d x_{2} \cdots d x_{k} .
$$

We shall denote the vector $\left(x_{1}, x_{2}, \cdots, x_{k}\right)$ by $\mathbf{x}$. Numerical methods for the evaluation of $I$ involve the calculation of $f(\mathbf{x})$ at a number $N$ of points $\mathbf{x}_{i}$. The most desirable of such methods for use on an electronic computer are those which require the evaluation of $f(\mathbf{x})$ at the smallest number of points $\mathbf{x}_{i}$ in order to obtain an estimate with a sufficiently small error.

The Monte Carlo method gives as an estimate for $I$ the sum

$$
\frac{1}{N} \sum_{i=1}^{N} f\left(\mathbf{x}_{i}\right)
$$

where the points $\mathbf{x}_{i}$ are chosen at random in the range of integration. The error of such an estimate has standard deviation $O\left(N^{-1 / 2}\right)$ provided that the function $f(\mathbf{x})$ satisfies certain conditions. It is sufficient that the function be bounded.

A Gauss-type formula for a one-dimensional integral takes the form

$$
\int_{a}^{a+h} g(y) d y=h \sum_{i=1}^{\nu} c_{i} g\left(a+\alpha_{i} h\right)+R .
$$

Received October 31, 1960. 
The remainder $R$ is $O\left(h^{2 v}\right)$ as $h$ varies for functions $g(y)$ satisfying certain conditions. We may estimate the integral

$$
\int_{a_{1}}^{a_{1}+h} \int_{a_{2}}^{a_{2}+h} \cdots \int_{a_{k}}^{a_{k}+h} f(\mathbf{x}) d x_{1} d x_{2} \cdots d x_{k}
$$

by applying the formula in each dimension, obtaining the direct product estimate

$$
h^{k} \sum_{i_{1}=1}^{\nu} \cdots \sum_{i_{k}=1}^{\nu} c_{i_{1}} \cdots c_{i_{k}} f\left(a_{1}+\alpha_{i_{1}} h, \cdots, a_{k}+\alpha_{i_{k}} h\right) .
$$

This gives the value of the integral with an error which is $O\left(h^{k+2 v-1}\right)$ as $h$ varies, provided that the function $f(x)$ possesses partial derivatives of order up to $2 \nu$ with respect to each variable and that these derivatives are bounded. These conditions are very strong in that $f(\mathbf{x})$ must have the derivative

$$
\frac{\partial^{2 \nu k} f}{\partial x_{1}^{2 \nu} \partial x_{2}^{2 \nu} \cdots \partial x_{k}^{2 \nu}},
$$

but they are probably more than is necessary.

If we divide the hypercube $0 \leqq x_{j} \leqq 1$ into $n^{k}$ smaller cubes with side $h=1 / n$, then using the $\nu$-point Gauss formula we obtain a formula for $I$ which requires $N=(m)^{k}$ values of $f(\mathbf{x})$. This gives the value of $I$ with an error which is

$$
O\left(n^{-2 v+1}\right)=O\left(N^{(-2 v+1) / k}\right)
$$

for fixed $\nu$ and $k$. Thus if $2 \nu-1<\frac{1}{2} k, \nu$ and $k$ being fixed, the error will not decrease as $N$ increases so rapidly as in the Monte Carlo method. This suggests that $\nu$ should be chosen large. However, it is not desirable to use a high-order Gauss formula since large errors may then arise if high-order partial derivatives of the function are large or if the function does not possess such derivatives. The highorder partial derivatives are large if the function has singularities in the $2 k$-dimensional complex domain near the region of integration.

Similar arguments to those above may be applied to multidimensional integration formulas obtained as the direct product of rules such as Simpson's rule, which gives an error that is $O\left(N^{-4 / k}\right)$. They may in fact be applied to any case where the domain of integration is divided into smaller regions as above.

In this connection the following two integration formulas should be mentioned.

(i) $\frac{1}{(2 h)^{k}} \int_{-h}^{h} \cdots \int_{-h}^{h} f(\mathbf{x}) d x_{1} \cdots d x_{k}=\left(1-\frac{1}{3} k\right) f(0,0, \cdots, 0)$

$$
\begin{aligned}
+\frac{1}{6}\{f(h, 0, \cdots, 0)+f(-h, 0, & \cdots, 0)+f(0, h, 0, \cdots, 0) \\
& +f(0,-h, 0, \cdots, 0)+\cdots\}+O\left(h^{4}\right) .
\end{aligned}
$$

(ii) $\frac{1}{(2 h)^{k}} \int_{-h}^{h} \cdots \int_{-h}^{h} f(\mathbf{x}) d x_{1} \cdots d x_{k}=\frac{2}{3} f(0,0, \cdots, 0)$

$$
\begin{aligned}
+\frac{1}{3.2^{k}}\{f(h, h, \cdots, h)+f(-h, h, \cdots, h) & +f(h,-h, h, \cdots, h) \\
& +f(-h,-h, h, \cdots, h)+\cdots\}+O\left(h^{4}\right) .
\end{aligned}
$$


Formula (i) requires $(2 k+1)$ values of $f(\mathbf{x})$ to estimate the integral over the hypercube of side $2 h$, and formula (ii) requires $\left(2^{k}+1\right)$ values. However, if the hypercube $0 \leqq x_{j} \leqq 1$ is divided into $n^{k}$ smaller ones of side $2 h$ then to evaluate $I$ by using (i) requires $(k+1) n^{k}+O\left(n^{k-1}\right)$ values of $f(\mathbf{x})$ and by using (ii) requires $2 n^{k}+O\left(n^{k-1}\right)$ values. Simpson's rule used in this way requires $(k+2) n^{k}+$ $O\left(n^{k-1}\right)$ values. Thus, for fixed $k$ the formulas (i) and (ii) and Simpson's rule give errors which are $O\left(N^{-4 / k}\right)$, but (ii) is the most efficient.

In this paper we shall describe methods which give errors which are $O\left(N^{-1}\right)$, $O\left(N^{-2}\right), O\left(N^{-3}\right)$ and $O\left(N^{-4}\right)$ when applied in any number of dimensions to functions which satisfy appropriate conditions. The points $\mathbf{x}_{i}$ at which the values of the function $f(\mathbf{x})$ are required can be calculated by a computer more easily than the random numbers required for the Monte Carlo method. No coefficients, such as are required in the case of the Gauss-type methods, need be calculated, nor is it necessary to decide in advance the number of points to be used.

In the following sections we first describe the methods as applied to the integration of functions periodic in each of the variables. In Section 3 we describe calculations leading to numerical estimates of some of the errors and to suitable sets of points $\mathbf{x}_{i}$ at which to calculate the function. In Section 4 we describe the practical application of the methods to non-periodic functions, and in Section 5 we give an example.

\section{The Integration of Periodic Functions.}

2.1. General Theory. Let $f\left(x_{1}, x_{2}, \cdots x_{k}\right)=f(\mathbf{x})$ be a periodic function with period $2 \pi$ in each of the $k$ variables $x_{1}, x_{2}, \cdots, x_{k}$. We shall describe a number of methods for estimating the integral

$$
I=\frac{1}{(2 \pi)^{k}} \int_{-\pi}^{\pi} \int_{-\pi}^{\pi} \cdots \int_{-\pi}^{\pi} f(\mathbf{x}) d x_{1} d x_{2} \cdots d x_{k} .
$$

These estimates will be based on sums of the type

$$
s(N)=\sum_{m} c_{N m} f\left(2 \pi m \alpha_{1}, 2 \pi m \alpha_{2}, \cdots, 2 \pi m \alpha_{k}\right),
$$

where the $\alpha_{i}$ are certain linearly independent irrational numbers and the $c_{N m}$ are certain coefficients chosen so that $s(N) \rightarrow I$ as $N \rightarrow \infty$. We shall be particularly concerned with sets of coefficients $c_{N m}$ such that the number of non-zero coefficients in the sum (1) is finite and of order $N$. We shall give estimates for the difference $s(N)-I$ in terms of $N$ and of bounds for the derivatives of $f(\mathbf{x})$.

Certain formulas which are particularly simple to use are based on sums $s(N)$ which can be expressed in terms of the repeated sums

$$
S_{1}(N)=\sum_{m=-N}^{N} f(2 \pi m \alpha)
$$

and

$$
S_{r}(N)=\sum_{m=0}^{N} S_{r-1}(m)
$$


where $r \geqq 2$. The $S_{r}(N)$ are modified Césaro means of the sequence $f(2 \pi m \boldsymbol{\alpha})$, terms with positive and negative $m$ being taken together. We shall show that the means

$$
s_{1}(N)=\frac{1}{2 N+1} S_{1}(N)
$$

and

$$
s_{2}(N)=\frac{1}{(N+1)^{2}} S_{2}(N),
$$

which are of the form (1), give good estimates of $I$ for large $N$. However, although for functions satisfying certain conditions the estimate for the error $s_{2}(N)-I$ is better than the estimate for $s_{1}(N)-I$, we find that the higher-order Césaro means do not give better estimates of $I$. We shall show that if $f(\mathbf{x})$ satisfies certain conditions then the means

$$
s_{3}(N)=\frac{1}{(N+1)^{2}(2 N+3)}\left\{S_{3}(2 N+1)-2 S_{3}(N)\right\}
$$

and

$$
s_{4}(N)=\frac{1}{(N+1)^{4}}\left\{S_{4}(2 N)-4 S_{4}(N-1)\right\}
$$

give substantially improved estimates of the integral. Under these conditions we shall show that for large $N$,

$$
s_{r}(N)-I=O\left(N^{-r}\right)
$$

for $r=1,2,3$ and 4 .

In the theory of the general method we are led to the consideration of the function

$$
k_{N}(\theta)=\sum_{m} c_{N m} e^{i m \theta},
$$

in terms of which we shall give estimates of the error. If for a particular set of coefficients $c_{N m}$,

$$
\left|k_{N}(\theta)\right| \leqq \frac{K}{\left|N \sin \frac{1}{2} \theta\right|^{r}}
$$

for some fixed constant $K=K(r)$ and for all $N$ and $\theta$, we shall say that the method has order $r$ with constant $K$. For the sums $s_{1}(N), s_{2}(N), s_{3}(N)$ and $s_{4}(N)$ the corresponding functions $k_{N}(\theta)$ are

$$
\begin{aligned}
& k_{N}^{(1)}(\theta)=\frac{1}{(2 N+1)} \frac{\sin \left(N+\frac{1}{2}\right) \theta}{\sin \frac{1}{2} \theta}, \\
& k_{N}^{(2)}(\theta)=\frac{1}{(N+1)^{2}} \frac{\sin ^{2} \frac{1}{2}(N+1) \theta}{\sin ^{2} \frac{1}{2} \theta}, \\
& k_{N}^{(3)}(\theta)=\frac{1}{(N+1)^{2}(2 N+3)} \frac{\sin ^{2} \frac{1}{2}(N+1) \theta \sin \left(N+\frac{3}{2}\right) \theta}{\sin ^{2} \frac{1}{2} \theta}
\end{aligned}
$$


and

$$
k_{N}^{(4)}(\theta)=\frac{1}{(N+1)^{4}} \frac{\sin ^{4} \frac{1}{2}(N+1) \theta}{\sin ^{4} \frac{1}{2} \theta} .
$$

Hence these sums give methods of order 1, 2, 3 and 4 respectively. We shall prove that a method of order $r$ gives an error which is $O\left(N^{-r}\right)$ provided that the integrand satisfies certain conditions.

A method may have order $r$ for several different values of $r$. In particular, if

$$
c_{N m}=c_{0} e^{-m^{2} / 2 N^{2}},
$$

where the constant $c_{0}$ is chosen so that

$$
\sum_{m} c_{N m}=1
$$

it follows from the theory of elliptic modular functions that the method is of order $r$ for all positive $r$. However, this method has certain disadvantages in practice.

2.2. The Numbers $\alpha_{i}$. We shall now prove a lemma which will enable us to prove that there exist sets of irrational numbers $\alpha_{i}$ which may be used to obtain good estimates of integrals. This lemma is a standard result in the theory of Diophantine approximation but we include the proof here for completeness. The lemma also shows that for most sets of numbers $\alpha_{i}$ the error is not substantially worse than in the estimates given. We show in Section 3 how suitable sets of numbers $\alpha_{i}$ may be found.

We shall denote a set of integers $\left(n_{1}, n_{2}, \cdots, n_{k}\right)$ by $\mathbf{n}$ and we shall write the scalar product $\left(n_{1} \alpha_{1}+n_{2} \alpha_{2}+\cdots+n_{k} \alpha_{k}\right)$ in the form $\mathbf{n} \cdot \boldsymbol{\alpha}$.

Lemma. Let $\phi(n)$ be any positive function such that

$$
\sum_{-\infty}^{\infty} 1 / \phi(n)=1
$$

Then there exist irrational numbers $\alpha_{1}, \alpha_{2}, \cdots, \alpha_{k}$ such that

$$
\phi\left(n_{1}\right) \phi\left(n_{2}\right) \cdots \phi\left(n_{k}\right)|\mathbf{n} \cdot \boldsymbol{\alpha}-n| \leqq 1 /(k+1)
$$

for all sets of integers $n_{1}, n_{2}, \cdots, n_{k}, n$ not all zero. Further, the measure of the set of $\alpha_{1}, \alpha_{2}, \cdots, \alpha_{k}$ with $0 \leqq \alpha_{i} \leqq 1$ such that

$$
\operatorname{lbd} \phi\left(n_{1}\right) \phi\left(n_{2}\right) \cdots \phi\left(n_{k}\right)|\mathbf{n} \cdot \boldsymbol{\alpha}-n| \leqq \delta /(k+1)
$$

is less than $\delta$.

We may suppose without loss of generality that $0 \leqq \alpha_{i} \leqq 1$. We observe that for fixed $\mathrm{n}, n$ the measure of the set of $\alpha$ such that

$$
\phi\left(n_{1}\right) \phi\left(n_{2}\right) \cdots \phi\left(n_{k}\right)|\mathbf{n} \cdot \boldsymbol{\alpha}-n| \leqq \delta /(k+1)
$$

is less than or equal to

$$
\frac{\delta}{(k+1)} \frac{1}{\max \left|n_{i}\right|} \frac{1}{\phi\left(n_{1}\right) \phi\left(n_{2}\right) \cdots \phi\left(n_{k}\right)} .
$$

But $n$ is a non-negative integer less than or equal to $k \max \left|n_{i}\right|$. Hence the measure 
of the set of $\boldsymbol{\alpha}$ which satisfy (4) for given $\mathrm{n}$ and some $n$ is less than or equal to

$$
\delta / \phi\left(n_{1}\right) \phi\left(n_{2}\right) \cdots \phi\left(n_{k}\right) .
$$

Thus the measure of the set of $\alpha$ which satisfy (3) is less than

$$
\delta \sum_{-\infty}^{\infty} \cdots \sum_{-\infty}^{\infty} \frac{1}{\phi\left(n_{1}\right) \phi\left(n_{2}\right) \cdots \phi\left(n_{k}\right)}=\delta
$$

(we have strict inequality since the regions overlap). Taking $\delta=1$ we obtain the first part of the lemma.

2.3. Estimates for the Error in the Integration of Periodic Functions. We now consider the problem of estimating the integral of a periodic function of $k$ variables which can be represented by an absolutely convergent multiple Fourier series

$$
f(\mathbf{x})=\sum_{n_{1}} \cdots \sum_{n_{k}} a_{n_{1} n_{2} \cdots n_{k}} e^{i \mathbf{n} \cdot \mathbf{x}} .
$$

We shall suppose that for some positive fixed number $s$ there exists a constant $M_{8}$ such that if none of the $n_{i}=0$,

$$
\left|a_{n_{1} n_{2}} \cdots n_{k}\right| \leqq M_{8}\left|n_{1} n_{2} \cdots n_{k}\right|^{-8} \text {. }
$$

We suppose further that if any of the $n_{i}=0$ the same inequality holds with the zero factors omitted from the denominator on the right hand side.

Since the Fourier series is absolutely convergent we may treat the contributions to the sum $s(N)$ from the individual terms of the Fourier series separately. We obtain

$$
\begin{aligned}
s(N) & =\sum_{m} c_{N m} f(2 \pi m \boldsymbol{\alpha}) \\
& =\sum_{n_{1}} \cdots \sum_{n_{k}} a_{n_{1} n_{2} \cdots n_{k}} k_{N}(2 \pi \mathbf{n} \cdot \boldsymbol{\alpha}) .
\end{aligned}
$$

Now $a_{0,0}, \ldots, 0=I$, which is the integral which we wish to evaluate. We shall suppose that the coefficients $c_{N m}$ are scaled so that $k_{N}(0)=1$. Then

$$
s(N)-I=\sum{ }^{\prime} a_{n_{1} n_{2} \cdots n_{k}} k_{N}(2 \pi \mathbf{n} \cdot \boldsymbol{\alpha}),
$$

where the prime' means that the term $(0,0, \cdots, 0)$ is omitted.

We suppose that the method is of order $r$ so that there exists a constant $K$ such that

$$
\left|k_{N}(\theta)\right| \leqq K\left|N \sin \frac{1}{2} \theta\right|^{-r}
$$

for all $N$ and $\theta$. Now

$$
|\sin \pi \xi| \geqq 2\|\xi\|
$$

where $\|\xi\|$ denotes the distance of $\xi$ from the nearest integer. Thus if the numbers $\alpha_{i}$ satisfy the condition (2) we have

$$
|\sin \pi \mathbf{n} \cdot \boldsymbol{\alpha}| \geqq \frac{2}{k+1} \frac{1}{\phi\left(n_{1}\right) \phi\left(n_{2}\right) \cdots \phi\left(n_{k}\right)} .
$$

Thus if we apply the bound for the Fourier coefficient and the condition (7) we 
deduce that the contribution of the term $n_{1}, n_{2}, \cdots, n_{k}$ to the sum is less than

$$
K M_{s} 2^{-r}(k+1)^{r}\left\{\phi\left(n_{1}\right) \phi\left(n_{2}\right) \cdots \phi\left(n_{k}\right)\right\}^{r}\left|n_{1} n_{2} \cdots n_{k}\right|^{-s} N^{-r} .
$$

We have supposed that we may drop any of the $n_{i}$ from the denominator if it is zero. If we suppose that the sum

$$
\sum^{\prime}\{\phi(n)\}^{r}|n|^{-8}
$$

converges we deduce that there exists a constant $C_{r, 8}$ depending only on $r$ and $s$ such that

$$
|s(N)-I| \leqq K M_{s} 2^{-r}(k+1)^{r} C_{r, 8}^{k} N^{-r} .
$$

We have the bound

$$
C_{r, 8} \leqq\{\phi(0)\}^{r}+\sum_{-\infty}^{\infty}\{\phi(n)\}^{r}|n|^{-8}
$$

for the coefficients $C_{r, 8}$. If $s>r+1$ we can choose an appropriate function $\phi(n)$ which leads to a numerical bound for the error of the method. However, this bound for $C_{r, 8}$ is by no means the best possible and we shall show how it may be improved.

We shall now prove the theorem.

TheOREM. If the numbers $\alpha_{1}, \alpha_{2}, \cdots, \alpha_{k}$ are chosen appropriately and if the function $f\left(x_{1}, x_{2}, \cdots, x_{k}\right)$ is periodic with period $2 \pi$ in each of the variables $x_{i}$ and its Fourier coefficients satisfy the condition (5) then if we apply any method of order $r<s$, where $r \geqq 1$, with constant $K$ there exist numbers $C_{r, s}$ such that

$$
|s(N)-I| \leqq K M_{8} 2^{-r}(k+1)^{r} A_{r, s, k} C_{r, 8}^{k} N^{-r} \text {. }
$$

The numbers $C_{r, 8}$ depend only on the numbers $\alpha_{i}$ and not on the particular method or the function to be integrated.

If $r=s$ it can be proved that $|s(N)-I|=O\left(N^{-r+\epsilon}\right)$ for $\epsilon>0$, but we shall not give the proof here.

In order to prove the theorem we suppose that the function $\phi(n)$ is chosen to be monotonic increasing for $n \geqq 0$ and such that $\phi(-n)=\phi(n)$. We divide the range of summation of the variables $n_{1}, n_{2}, \cdots, n_{k}$ into zones defined by

$$
N_{i}<n_{i} \leqq 2 N_{i}
$$

(or if $n_{i}$ is negative $-2 N_{i} \leqq n_{i}<-N_{i}$; zones for which some of the variables are zero are also allowed). Now if $\mathbf{n}^{\prime}$ and $\mathbf{n}^{\prime \prime}$ are two sets of numbers in the same zone we deduce that $\left|n_{i}^{\prime}-n_{i}^{\prime \prime}\right| \leqq N_{i}$. Hence

$$
\begin{aligned}
\left\|\left(\mathbf{n}^{\prime}-\mathbf{n}^{\prime \prime}\right) \cdot \boldsymbol{\alpha}\right\| & \geqq 1 /(k+1) \phi\left(n_{1}{ }^{\prime}-n_{1}^{\prime \prime}\right) \phi\left(n_{2}{ }^{\prime}-n_{2}{ }^{\prime \prime}\right) \cdots \phi\left(n_{k}{ }^{\prime}-n_{k}{ }^{\prime \prime}\right) \\
& \geqq 1 /(k+1) \phi\left(N_{1}\right) \phi\left(N_{2}\right) \cdots \phi\left(N_{k}\right)
\end{aligned}
$$

so that

$$
\left|\left\|\mathbf{n}^{\prime} \cdot \boldsymbol{\alpha}\right\|-\left\|\mathbf{n}^{\prime \prime} \cdot \boldsymbol{\alpha}\right\|\right| \geqq 1 /(k+1) \phi\left(N_{1}\right) \phi\left(N_{2}\right) \cdots \phi\left(N_{k}\right) .
$$

We deduce that the number of sets of numbers $\mathbf{n}$ in the zone with

$$
\|\mathbf{n} \cdot \boldsymbol{\alpha}\| \leqq \nu /(k+1) \phi\left(N_{1}\right) \phi\left(N_{2}\right) \cdots \phi\left(N_{k}\right)
$$


is at most $2 \nu+2$. Now for any set of numbers $n$,

$$
\|\mathbf{n} \cdot \boldsymbol{\alpha}\| \geqq 1 /(k+1) \phi\left(n_{1}\right) \boldsymbol{\phi}\left(n_{2}\right) \cdots \boldsymbol{\phi}\left(n_{k}\right)
$$

so that the contribution of that set to the sum (6) is less than or equal to

$$
2^{-r}(k+1)^{r} M_{s} K\left\{\phi\left(n_{1}\right) \phi\left(n_{2}\right) \cdots \phi\left(n_{k}\right)\right\}^{r}\left|n_{1} n_{2} \cdots n_{k}\right|^{-s} N^{-r} .
$$

If we assume that $\{\phi(n)\}^{r}|n|^{-s}$ decreases monotonically for $n>0$ we deduce that the contribution of this term to the sum is less than

$$
2^{-r}(k+1)^{r} M_{s} K\left\{\phi\left(N_{1}\right) \phi\left(N_{2}\right) \cdots \phi\left(N_{k}\right)\right\}^{r}\left|N_{1} N_{2} \cdots N_{k}\right|^{-s} N^{-r} \text {. }
$$

Thus, the sum of the contributions of all terms in the zone $N_{i}<n_{i} \leqq 2 N_{i}$ to the sum (6) is less than

$$
\begin{aligned}
2^{-r}(k+1)^{r} M_{s} K\left\{\phi\left(N_{1}\right) \phi\left(N_{2}\right) \cdots \phi\right. & \left.\left(N_{k}\right)\right\}^{r} \\
& \cdot\left|N_{1} N_{2} \cdots N_{k}\right|^{-8}\left(2+2 \sum_{\nu=1}^{N_{1} N_{2} \cdots N_{k}} \nu^{-r}\right) N^{-r} .
\end{aligned}
$$

Similar results hold for those zones for which some of the $n_{i}$ are negative or zero.

If $s>r$ and $r>1$ we may choose $\phi(n)$ so that the sum over all zones converges. We obtain the inequality

$$
|s(N)-I| \leqq 2^{-r}(k+1)^{r} M_{s} K\{2 \zeta(r)+2\}\left(\{\phi(0)\}^{r}+2 \sum{ }^{\prime}\left\{\phi\left(N_{1}\right)\right\}^{r} N_{1}^{-s}\right)^{k} N^{-r},
$$

where $N_{1}$ runs through the powers of 2 and $\zeta(r)$ is the Riemann zeta function. This inequality is of the form (8) with $A_{r, s, k}=\{2 \zeta(r)+2\}$.

If $r=1$ and $s>1$ we obtain an expression with a different $C_{r, s}$ and a coefficient $A_{r, s, k}$ depending on $s$ and $k$.

These estimates for the errors are very crude but give some indication of the power of the methods. In the next section we shall describe how sets of numbers $\alpha_{i}$ can be obtained and how numerical bounds for the errors in the estimates of the integrals can be calculated.

\section{Numerical Estimates for the Error.}

3.1. A Method for Obtaining Numerical Estimates. In this section we shall be especially concerned with the methods using the sums $s_{2}(N)$ and $s_{4}(N)$ to estimate the integral of a periodic function

$$
f(\mathbf{x})=f\left(x_{1}, x_{2}, \cdots, x_{k}\right)=\sum_{n_{1}} \cdots \sum_{n_{k}} a_{n_{1} n_{2} \cdots n_{k}} e^{i \mathbf{n} \cdot \mathbf{x}} .
$$

Note that this function has period 2 in each of the variables $x_{i}$. As before, we shall assume that the Fourier coefficients satisfy an inequality

$$
\left|a_{n_{1} n_{2}} \cdots n_{k}\right| \leqq M_{s}\left|n_{1} n_{2} \cdots n_{k}\right|^{-s} \text {. }
$$

We shall be particularly concerned with the cases $s=2$ and $s=4$. In order to obtain a convenient form for the final results we shall replace zero factors in the denominator of the right-hand side of $(9)$ by $\left(6 / \pi^{2}\right)$ when $s=2$ and by $\left(360 / 7 \pi^{4}\right)$ when $s=4$. 
We recall that the functions $k_{N}(\theta)$ for $r=2$ and $r=4$ take the form

$$
k_{N}^{(2)}(\theta)=\frac{1}{(N+1)^{2}} \frac{\sin ^{2} \frac{1}{2}(N+1) \theta}{\sin ^{2} \frac{1}{2} \theta}
$$

and

$$
k_{N}^{(4)}(\theta)=\frac{1}{(N+1)^{4}} \frac{\sin ^{4} \frac{1}{2}(N+1) \theta}{\sin ^{4} \frac{1}{2} \theta} .
$$

Both these functions are positive.

Thus

$$
\begin{aligned}
\left|s_{r}(N)-I\right| & =\sum \cdots \sum{ }^{\prime} a_{n_{1} n_{2} \cdots n_{k}} k_{N}^{(r)}(\pi \mathbf{n} \cdot \boldsymbol{\alpha}) \\
& \leqq M_{8} \sum \cdots \sum\left|n_{1} n_{2} \cdots n_{k}\right|^{-8} k_{N}^{(r)}(\pi \mathbf{n} \cdot \boldsymbol{\alpha})
\end{aligned}
$$

We now define the functions

$$
\begin{aligned}
f_{s}(\mathbf{x}) & =M_{8}^{*} \sum \cdots \sum\left|n_{1} n_{2} \cdots n_{k}\right|^{-8} e^{\pi i(\mathbf{n} \cdot \mathbf{x})} \\
& =M_{8}^{*} \prod_{j=1}^{k}\left(\sum_{n=-\infty}^{\infty}|n|^{-8} e^{\pi i n x_{j}}\right)
\end{aligned}
$$

where $M_{s}{ }^{*}$ is chosen so that $f_{s}(0,0, \cdots, 0)=1$ and where, when $n=0$, the factor $n^{-8}$ is replaced by the appropriate fraction for $s=2$ or $s=4$.

By summation of the trigonometric series we deduce that

$$
f_{2}(\mathbf{x})=\prod_{i=1}^{k}\left\{\left(1-\left|x_{i}\right|\right)^{2}\right\}
$$

and

$$
f_{4}(\mathbf{x})=\prod_{i=1}^{k}\left\{2-\left(1-\left|x_{i}\right|\right)^{2}\right\}\left(1-\left|x_{i}\right|\right)^{2} .
$$

We denote the values of the sums $s_{r}(N)$ corresponding to the functions $f_{8}(\mathbf{x})$ by $s_{r, s}(N)$, and the mean value of $f_{s}(\mathbf{x})$ over the periodic cell by $I_{s}$.

Then we deduce that

$$
\left|s_{r}(N)-I\right| \leqq\left(M_{s} / M_{s}^{*}\right)\left(s_{r, 8}(N)-I_{8}\right) .
$$

Thus, we may say that the functions $f_{2}(\mathbf{x})$ and $f_{4}(\mathbf{x})$ are the worst functions for numerical integration whose coefficients satisfy the inequality $(\dot{9})$ with $s=2$ and $s=4$.

If, therefore, we find sets of numbers $\alpha_{i}$ which make $\left(s_{r, s}(N)-I_{s}\right)$ for a practical range of $N$ as small as possible, these $\alpha_{i}$ will give good estimates for the integral of any periodic function whose Fourier coefficients satisfy (9).

3.2. The Determination of the $\alpha_{i}$. The Ferranti Mercury computer at Manchester University was used to find good sets of $\alpha_{i}$ for $(r, s)=(2,2)$ and $(2,4)$. This was done by minimizing the upper bounds of $(N+1)^{2}\left\{s_{r, s}(N)-I_{s}\right\}$ for $0<N \leqq N_{1}$. The minimization was performed by a random walk in the $\alpha$-space, a step being taken only if it decreased the upper bound. The size of the step was 
reduced when the upper bound could not be decreased otherwise and the number $N_{1}$ was increased when the step was reduced.

For $s=2, I_{s}=\left(\frac{1}{3}\right)^{k}$ and for $s=4, I_{s}=\left(\frac{15}{7}\right)^{k}$. In both cases

$$
f(0,0, \cdots, 0)=1
$$

and $f(\mathbf{x}) \geqq 0$ for all $\mathbf{x}$, so that $S_{1}(m) \geqq 1$ and $S_{2}(N) \geqq N+1$. Hence, for $s=2$ we have

$$
(N+1)^{2}\left\{S_{2,2}(N)-I_{2}\right\} \geqq(N+1)-(N+1)^{2}\left(\frac{1}{3}\right)^{k} .
$$

For a certain value $N_{m}$ of $N$ the right-hand side of this inequality approaches its maximum value of $\frac{1}{4} \cdot 3^{k}$. Therefore, for values of $N_{1}>N_{m}$ we cannot expect the upper bound lub $(N+1)^{2}\left\{s_{2,2}(N)-I_{2}\right\}$ to be less than $\frac{1}{4} \cdot 3^{k}$. The corresponding value for $s=4$ is $\frac{1}{4}\left(\frac{15}{7}\right)^{k}$.

It was found that the points $\alpha$ converged to points for which the upper bound was reasonably small. In Figure 1 the logarithms of the upper bounds for $s=2$ and $s=4$ are plotted against $k$. For $s=2, N_{1}$ was taken up to 1500 ; for $s=4$, up to 1000 . The values $\frac{1}{4} \cdot 3^{k}$ for $s=2$ and $\frac{1}{4}\left(\frac{15}{7}\right)^{k}$ for $s=4$ are shown on the same logarithmic graph as straight lines. The point for $s=2, k=8$ lies below the appropriate line; for this case $N_{1}$ was less than the number $N_{m}$. The numbers $\alpha_{i}$ obtained for $s=2$ and $s=4$ are given in Tables 1 and 2 respectively. It should be realized that although these are good sets of $\alpha_{i}$ they are not claimed to be the best possible.

The convergence of the points $\alpha$ suggests that there may be limit points $\boldsymbol{\alpha}^{\prime}$ such that the bounds

$$
\operatorname{lub}_{0<N<\infty}(N+1)^{s}\left\{s_{r, 8}(N)-I_{s}\right\}
$$

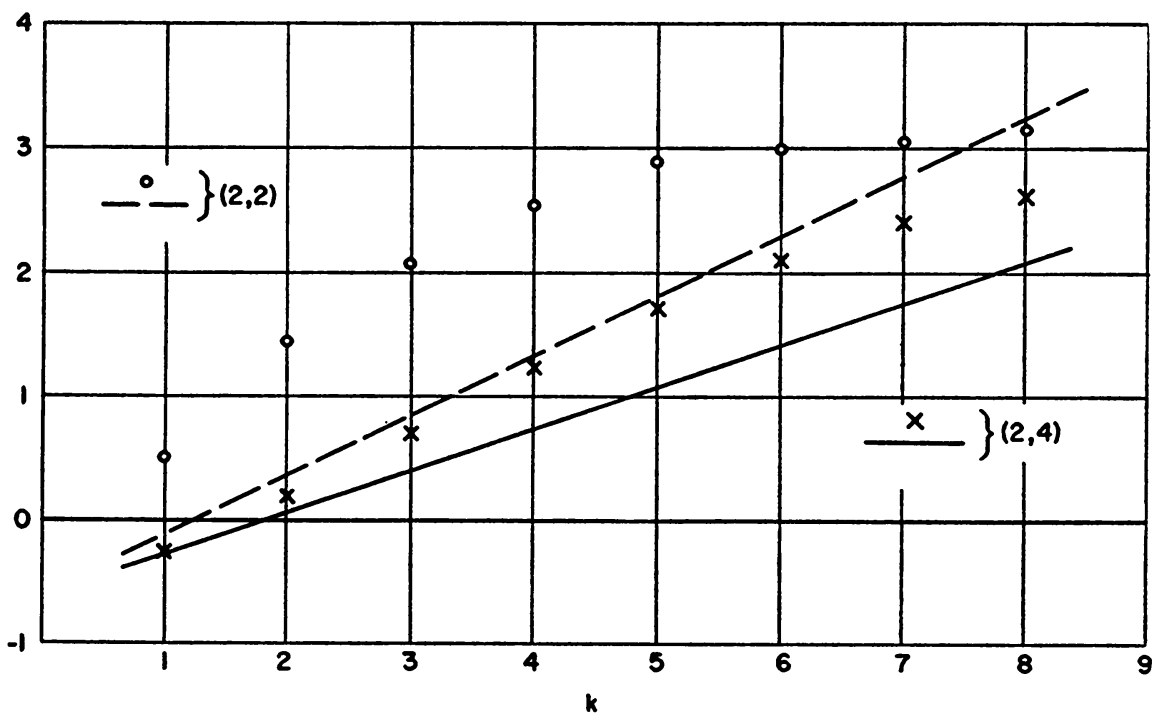

FIG. 1.-Errors associated with the numbers $\alpha_{i}$ for $(r, s)=(2,2)$ and $(2,4)$. The points are the logarithms to base 10 of the computed errors and the lines are those below which, for large enough $N$, the points cannot be expected to fall. 
TABLE 1

Numbers $\alpha_{i}$ for $(r, s)=(2,2)$

\begin{tabular}{c|c|c}
\hline$k=1$ & $k=5$ & $k=7$ \\
0.73258893 & 0.95734608 & 0.80638723 \\
$k=2$ & 0.86730270 & 0.22584927 \\
0.62055505 & 0.09724025 & 0.72510075 \\
0.22610245 & 0.31301950 & 0.51310685 \\
$k=3$ & 0.48476582 & 0.11080509 \\
0.96498949 & $k=6$ & 0.60161858 \\
0.81091316 & 0.43657951 & 0.92715171 \\
0.46960090 & 0.59185199 & $k=8$ \\
$k=4$ & 0.05024400 & 0.73750248 \\
0.62366851 & 0.84373919 & 0.08314415 \\
0.04150108 & 0.38104000 & 0.84753682 \\
0.48574769 & 0.75808683 & 0.88989711 \\
0.27210703 & & 0.80254484 \\
& & 0.27951501 \\
& & 0.67340402 \\
\hline
\end{tabular}

for these points are not substantially larger than the values given in the figure, but the author has not been able to prove this.

In the case $k=1, r=2, s=4$, however, he has been able to prove the following result.

If, for some $\alpha,(N+1)^{2}\left\{s_{2,4}(N)-I_{4}\right\}$ for $0 \leqq N \leqq N_{1}$ then there exists an irrational $\alpha^{\prime}$ such that for all $N$

$$
(N+1)^{2}\left\{s_{2,4}(N)-I_{4}\right\} \leqq 2 B\left\{1+O\left(N_{1}^{-1 / 8}\right)\right\}
$$

and such that $\left|\alpha^{\prime}-\alpha\right|=O\left(N_{1}^{-11 / 8}\right)$.

4. Application to the Integration of Non-Periodic Functions. We shall now show how the methods of Section 2 may be applied to obtain estimates of integrals of non-periodic functions of several variables. Suppose that it is required to evaluate the integral

$$
I=\iint \cdots \int_{R} F\left(x_{1}, x_{2}, \cdots, x_{k}\right) d x_{1} d x_{2} \cdots d x_{k}
$$

where $R$ is some region of the $k$-dimensional space and $F(\mathbf{x})$ is some given function. We shall show how under certain special circumstances it is possible to transform the problem to that of the integration of a periodic function.

We have seen that we can obtain good estimates for the error in the integration of a periodic function if its Fourier coefficients tend to zero rapidly. Now, the Fourier coefficients tend to zero rapidly if the function is continuous and possesses partial derivatives of high orders. We shall, therefore, attempt to construct "smooth" periodic functions. The methods given below for doing this are intended only as examples. It will be apparent that each problem may require special treatment to obtain the best results. Also, if the function $F(\mathbf{x})$ possesses unknown dis- 
TABLE 2

Numbers $\alpha_{i}$ for $(r, s)=(2,4)$

\begin{tabular}{c|c|c}
\hline$k=1$ & $k=5$ & $k=7$ \\
0.83969144 & 0.44810200 & 0.58505729 \\
$k=2$ & 0.53589831 & 0.50196855 \\
0.59734470 & 0.56039410 & 0.7779734 \\
0.92828094 & 0.83630131 & 0.60504620 \\
$k=3$ & 0.22148205 & 0.62193588 \\
0.74235492 & $k=6$ & 0.84244165 \\
0.57387033 & 0.10613747 & 0.64543976 \\
0.32279917 & 0.40278232 & $k=8$ \\
$k=4$ & 0.88772556 & 0.23975940 \\
0.17665781 & 0.43554826 & 0.01544979 \\
0.71327190 & 0.17219381 & 0.57794809 \\
0.98875216 & & 0.8182909 \\
0.60299793 & & 0.78068912 \\
& & 0.62319488 \\
& & 0.603890617 \\
\hline
\end{tabular}

continuities, it will not be possible to remove these from the periodic function constructed.

We first consider the integral

$$
I=\int_{0}^{1} \int_{0}^{1} \cdots \int_{0}^{1} F\left(x_{1}, x_{2}, \cdots, x_{k}\right) d x_{1} d x_{2} \cdots d x_{k} .
$$

This integral may be written in the form

$$
I=\frac{1}{2^{k}} \int_{-1}^{1} \int_{-1}^{1} \cdots \int_{-1}^{1} F\left(\left|z_{1}\right|,\left|z_{2}\right|, \cdots,\left|z_{k}\right|\right) d z_{1} d z_{2} \cdots d z_{k} .
$$

The function $f\left(z_{1}, z_{2}, \cdots, z_{k}\right)=F\left(\left|z_{1}\right|,\left|z_{2}\right|, \cdots,\left|z_{k}\right|\right)$ may be regarded as a periodic function with period 2 in each of the variables $z_{i}$. Then $f$ is continuous if $F$ is continuous, and if $F$ possesses a derivative

$$
\frac{\partial^{k} F}{\partial x_{1} \partial x_{2} \cdots \partial x_{k}}
$$

of bounded variation it may be proved that the Fourier coefficients of $f$ satisfy, for some $M_{2}$,

$$
\left|a_{n_{1} n_{2} \cdots n_{k}}\right| \leqq M_{2}\left|n_{1} n_{2} \cdots n_{k}\right|^{-2} .
$$

This implies that the means $s_{1}(N)$ and $s_{2}(N)$ will give estimates for $I$ with errors $O\left(N^{-1}\right)$ and $O\left(N^{-2+\epsilon}\right)$ respectively. These methods have the particular advantage that since $f(\mathbf{z})=f(-\mathbf{z})$ the means $s_{1}(N)$ and $s_{2}(N)$ may be evaluated from the values of $F$ at $N+1$ points. The sum $S_{1}(N)$ may be expressed in the form

$$
S_{1}(N)=F(0,0, \cdots, 0)+2 \sum_{n=1}^{N} F\left(2\left|\left\{\frac{1}{2} n \alpha_{1}\right\}\right|, \cdots, 2\left|\left\{\frac{1}{2} n \alpha_{k}\right\}\right|\right)
$$


where the brackets \{\} denote the fractional part, lying in the range $\left(-\frac{1}{2}, \frac{1}{2}\right)$. For such a function the basic points of Table 1 are suitable.

We now consider the application of higher-order methods to the integral

$$
I=\int_{-1}^{1} \int_{-1}^{1} \cdots \int_{-1}^{1} F\left(z_{1}, z_{2}, \cdots, z_{k}\right) d z_{1} d z_{2} \cdots d z_{k} .
$$

We make the substitution $z_{i}=P_{r}\left(x_{i}\right)$ where $r \geqq 1$ and

$$
P_{r}\left(x_{i}\right)=A_{r} \int_{0}^{x_{i}}\left(1-u^{2}\right)^{r} d u
$$

and $A_{r}$ is chosen so that $P_{r}( \pm 1)= \pm 1$. Then

$$
\begin{aligned}
I & =\int_{-1}^{1} \cdots \int_{-1}^{1} F\left(P_{r}\left(x_{1}\right), \cdots, P_{r}\left(x_{k}\right)\right) P_{r}^{\prime}\left(x_{1}\right) \cdots P_{r}^{\prime}\left(x_{k}\right) d x_{1} \cdots d x_{k} \\
& =\frac{1}{2^{k}} \int_{-1}^{1} \int_{-1}^{1} \cdots \int_{-1}^{1} f\left(x_{1}, x_{2}, \cdots, x_{k}\right) d x_{1} d x_{2} \cdots d x_{k},
\end{aligned}
$$

say. Then $f=0$ if any $x_{i}= \pm 1$, and $F$ is bounded. Thus if $F$ is continuous $f$ may be regarded as a continuous periodic function of the $x_{i}$. If $F$ possesses a partial derivative

$$
\frac{\partial^{k r} F}{\left(\partial x_{1} \partial x_{2} \cdots \partial x_{k}\right)^{r}}
$$

of bounded variation then so will $f$. It is then possible to obtain an estimate

$$
s_{r}(N)-I=O\left(N^{-r}\right)
$$

so that the application of a method of order $r$ will give an error of order $N^{-r}$ and the application of a method of order $r+1$ will give an error of order $N^{-r-1+\epsilon}$.

The numbers $\alpha_{i}$ of Table 2 can be used to calculate the sums $s_{2}(N)$ when the partial derivative $\partial^{3 k} f /\left(\partial x_{1} \partial x_{2} \cdots \partial x_{k}\right)^{3}$ of the function has bounded variation.

It is also possible to apply these methods to the more difficult problem of the evaluation of an integral taken over some more general regions. It is, of course, possible that there may exist some transformation of the variables which reduces the integral to periodic form. For example, in the evaluation of the integral of a function taken over the interior of a circle we may take polar coordinates. The function is then a periodic function of the angular variable. It may be made into a periodic function of the radius with derivatives of appropriate orders by one of the techniques above.

In the case of the integration of a function $F(x)$ over the infinite range $(-\infty, \infty)$ we may at once consider the periodic function

$$
\sum_{-\infty}^{\infty} F(x+2 \pi n)
$$

but this may mean that we have to take a very large number of points if $F(x)$ does not tend to zero rapidly as $x \rightarrow \pm \infty$.

A more general method is the method of extrusion. Suppose that a region $R$ with a smooth boundary is contained in a sphere $\mathrm{S}$ with center $\mathrm{O}$ in $\mathrm{R}$. Then, if $\mathrm{P}$ is 
any point in the sphere we may construct the line OP. If $\mathrm{R}$ is a star body about $\mathrm{O}$ this line will meet the boundary in one point $\mathrm{Q}$. We may then evaluate the function $F$ at a point $\mathrm{P}^{\prime}$ at a distance $\mathrm{OP} \cdot \mathrm{OQ} / r$ from $\mathrm{O}$ along the line $\mathrm{OP}$, where $r$ is the radius of the sphere. If this value is multiplied by $(\mathrm{OQ} / r)^{k}$ we obtain a function defined in the sphere which has the same integral over the sphere as $F$ has over $R$.

This process of extrusion may be generalized to the extrusion of any star body to any other star body containing it. It will generally be better to use a sphere rather than a cube if $R$ has a smooth boundary, otherwise discontinuities are introduced into the derivatives.

5. Exampla of Use. The method with the most practical application is probably that using the sum $s_{2}(N)$. The sum $s_{4}(N)$ may be more suitable if higher accuracy is required but for the results to be better than those for $s_{2}(N)$, the function $f$ must have higher-order partial derivatives.

The values of $s_{2}(N)$ can be calculated at chosen values of $N$ while the repeated sum $S_{2}(N)$ is being accumulated. The accuracy of the results can be estimated by examining the convergence of the successive values of $s_{2}(N)$.

A refinement may be introduced in order to decrease the rounding errors. The sum $S_{2}(N)$ becomes very large and for a floating point computer with a fixed number of significant figures the rounding error introduced at each addition is proportional to the larger of the two quantities added. Thus, the sum $s_{2}(N)$ will not be accurate to the full capacity of the computer. A method of overcoming this difficulty is to calculate an estimate $I_{n}{ }^{*}=S_{2}(N) /(N+1)^{2}$ at intervals in the computation, and to subtract this value from each subsequent calculation of the integrand, so that the accumulated sums are

and

$$
S_{1}^{*}(N)=\sum_{m=-N}^{N}\left\{f(m \boldsymbol{\alpha})-I_{n}^{*}\right\}
$$

$$
S_{2}^{*}(N)=\sum_{m=0}^{N} S_{1}^{*}(m)
$$

TABLE 3

Estimates for $\int_{0}^{1} \int_{0}^{1} \cdots \int_{0}^{1} e^{-x_{1} x_{3} \cdots x_{5}} d x_{1} d x_{2} \cdots d x_{5}$

\begin{tabular}{r|c|c|c}
\hline \multicolumn{1}{c|}{$N$} & $s_{2}(N)$ & $s_{1}(N)$ & Monte Carlo \\
\hline 1000 & 0.97062580 & 0.97062392 & 0.96763166 \\
2000 & 0.97063927 & 0.97082902 & 0.96870265 \\
3000 & 0.97066765 & 0.97054070 & 0.96885258 \\
4000 & 0.97066383 & 0.97068153 & 0.96944396 \\
5000 & 0.97065630 & 0.97065925 & 0.96950137 \\
6000 & 0.97065761 & 0.97061983 & 0.96990269 \\
7000 & 0.97065639 & 0.97068925 & 0.97018578 \\
8000 & 0.97065632 & 0.97064881 & 0.97030504 \\
9000 & 0.97065706 & 0.97063833 & 0.97038771 \\
10000 & 0.97065854 & 0.97066307 & 0.97032729 \\
11000 & 0.97065860 & 0.97065947 & 0.97029480 \\
12000 & 0.97065744 & 0.97067426 & 0.97048290 \\
\hline
\end{tabular}

Exact value $=0.97065719$ 
The sum $S_{2}{ }^{*}(N)$ does not then become very large. At each successive re-estimate $I_{n+1}^{*}=I_{n}{ }^{*}+S_{2}{ }^{*}(N) /(N+1)^{2}$ we replace the current $S_{2}{ }^{*}(N)$ by zero and the current $S_{1}^{*}(N)$ by $S_{1}^{*}(N)+(2 N+1)\left(I_{n}{ }^{*}-I_{n+1}^{*}\right)$. The same method used in a fixed-point computer will reduce the amount of scaling necessary.

As an example the integral

$$
\int_{0}^{1} \int_{0}^{1} \cdots \int_{0}^{1} e^{-x_{1} x_{2} \cdots x_{k}} d x_{1} d x_{2} \cdots d x_{k}
$$

was calculated on the Mercury computer. Some results for $k=5$ are given in Table 3 . We give the sum $s_{2}(N)$ for $N=1000(1000) 12000$. The method described above was used to reduce rounding errors, with the estimate $I_{n}{ }^{*}$ calculated at every 10th value of $N$ up to $N=100$ and every 100th value thereafter. For comparison, the sum $s_{1}(N)$ is also given, and an estimate using the ordinary Monte Carlo method with $N$ random sampling points.

6. Acknowledgments. The author is grateful for many helpful discussions with Dr. J. C. P. Miller, Dr. D. J. Wheeler, Mr. H. P. F. Swinnerton-Dyer, and others, and to the staff of the Manchester University Computing Machine Laboratory.

Department of Mathematics

The University

Manchester, 13, England

1. P. Davis \& P. Rabinowitz, "Some Monte Carlo experiments in computing multiple integrals," $M T A C$, v. 10,1956, p. $1-8$.

2. P. C. HAMmER, "Numerical evaluation of multiple integrals," On Numerical Approximation, ed. R. E. Langer. Proceedings of symposium, University of Wisconsin Press, Madison, 1959 , p. 99-115.

3. J. M. Hammersley \& K. W. Morton, "A new Monte Carlo technique: antithetic variates," Proc. Camb. Philos. Soc., v. 52, 1956, p. 449-475.

4. J. M. Hammersley \& J. G. Mauldon, "General principles of antithetic variates," Proc. Camb. Philos. Soc., v. 52, 1956, p. 476-481.

5. J. C. P. MILlER, "Numerical quadrature over a rectangular domain in two or more dimensions, Part 1. Quadrature over a square, using up to sixteen equally spaced points," Math. Comp., v. 14, 1960, p. 13-20.

6. J. C. P. Miller, "Numerical quadrature over a rectangular domain in two or more dimensions, Part 2. Quadrature in several dimensions, using special points," Math. Comp., v. 14, 1960, p. 130-138.

7. J. C. P. Miller, "Numerical quadrature over a rectangular domain in two or more dimensions, Part 3. Quadrature of a harmonic integral," Math. Comp., v. 14, 1960, p. 240-248. 